\title{
The Capacity for a Class of Fractal Functions
}

\author{
D. P. Hardin and P. R. Massopust \\ Georgia Institute of Technology, Atlanta, Ga 30332, USA
}

\begin{abstract}
We present a formula for the capacity of the graphs of certain fractal functions. We show that this formula can also be obtained using the Lyapunov exponents of an associated dynamical system.
\end{abstract}

Recently there has been great interest in the calculation of the Hausdorff dimension and the capacity (which are usually the same for compact sets) of fractals. These dimensions can often only be calculated numerically, however, there are sets whose scaling properties permit their explicit calculation (see [Mo, $\mathrm{Hu}, \mathrm{BaDe}, \mathrm{BaHa}, \mathrm{BeUr}, \mathrm{KaMaYo]})$.

In this note, we present a formula for the capacity of the graphs of certain fractal functions. Our result is related to the results of [BeUr, $\mathrm{KaMaYo,} \mathrm{BaHa].}$ Finally, we note that our formula can be obtained using the Lyapunov exponents of an associated dynamical system, supporting a conjecture of Yorke's.

\section{Preliminaries}

Let $K$ be a compact metric space or a closed subset of $\mathbb{R}^{n}$ with distance function $d$. Let $H$ be the set of all closed subsets of $K$. If we introduce the Hausdorff metric

$$
h(A, B)=\max \left\{\sup _{x \in A} \inf d(x, y), \sup _{x \in B} \inf _{y \in A} d(x, y)\right\},
$$

for all $A, B \in H$, on $H$, then $(H, h)$ is a complete metric space.

Let $w_{i}: K \supsetneq, \quad i=1, \ldots, N$, be continuous and contractive. Then $\left(K, w_{i}: i=1, \ldots, N\right)$ is called a hyperbolic iterated function system (h.i.f.s.) on $K$. Let $\underline{w}: H \triangleright$ be defined as $\underline{w}(A)=\bigcup_{i=1}^{N} w_{i}(A)$, for all $A \in H$, then $\underline{w}$ is a contraction on $H$ and thus possesses a unique fixed point. This fixed point is called the attractor for the h.i.f.s. $\left(K, w_{i}: i=1, \ldots, N\right)$ (see $\left.[\mathrm{Hu}, \mathrm{BaDe}, \mathrm{Ba}]\right) .\left(K, w_{i}: i=1, \ldots, N\right)$ admits a 
stationary measure $\mu$, the so-called $p$-balanced measure, given by

$$
\int_{\boldsymbol{K}} \phi d \mu=\sum_{i=1}^{N} p_{i} \int_{K} \phi \circ w_{i} d \mu
$$

for all $\phi \in C(K)$ and a set of probabilities $p=\left(p_{1}, \ldots, p_{N}\right)$ (see $\left.[\mathrm{BaDe}]\right)$.

Now let $K=[0,1] \times \mathbb{R}$ and let $\left\{\left(x_{i}, y_{i}\right): i=0, \ldots, N\right\}$ be $N+1$ interpolation points in $K$. We will restrict ourselves to the case of equal horizontal scalings $x_{i}-x_{i-1}=\frac{1}{N}, i=1, \ldots, N$.

We now define a h.i.f.s. $\left(K, w_{i}: i=1, \ldots, N\right)$ the following way

$$
w_{i}(x, y)=\left(\begin{array}{cc}
\frac{1}{N} & 0 \\
b_{i} & a_{i}
\end{array}\right)\left(\begin{array}{l}
x \\
y
\end{array}\right)+\left(\begin{array}{c}
\frac{i-1}{N} \\
k_{i}
\end{array}\right),
$$

where $b_{i}=y_{i}-y_{i-1}-a_{i}\left(y_{N}-y_{0}\right), k_{i}=y_{i-1}-a_{i} y_{0},\left|a_{i}\right|<1, i=1, \ldots, N$ and $\sum_{i}\left|a_{i}\right|>1$. Then the maps $w_{i}, i \in\{1, \ldots, N\}$, are contractive in the complete metric space $(K, \delta)$, where

$$
\delta\left(\left(u_{1}, u_{2}\right),\left(v_{1}, v_{2}\right)\right)=\left|u_{1}-u_{2}\right|+\frac{1-\frac{1}{N}}{2 \beta}\left|v_{1}-v_{2}\right|
$$

with $\beta>\max \left\{\left|b_{i}\right|: i=1, \ldots, N\right\},\left(u_{1}, u_{2}\right),\left(v_{1}, v_{2}\right) \in K$. Hence $\left(K, w_{i}: i=1, \ldots, N\right)$ possesses a unique attractor which is easily shown to be the graph of a continuous function $f: K \supseteq$ passing through $\left(x_{0}, y_{0}\right), \ldots,\left(x_{N}, y_{N}\right) . f$ is called a fractal interpolation function (f.i.f.) (for details and more general f.i.f.'s see [Ba]).

\section{Main Result}

Recall the capacity $C(S)$ of a bounded set $S$ is defined to be

$$
\lim _{\varepsilon \rightarrow 0} \frac{\log \mathscr{N}(\varepsilon)}{\log \varepsilon}, \quad \text { if it exists, }
$$

where $\mathscr{N}(\varepsilon)$ denotes the minimum number of $\varepsilon$ balls needed to cover $S$. Note that the limit remains unchanged if the continuous variable $\varepsilon$ is replaced by any sequence $\left\{\varepsilon_{n}\right\}$ with $\varepsilon_{n} \downarrow 0$ and

$$
\frac{\log \varepsilon_{n+1}}{\log \varepsilon_{n}} \rightarrow 1
$$

Let $G$ be the graph of a f.i.f. $f$ as defined above.

$$
\text { Let } \mathscr{C}=\left\{\left[\frac{k-1}{N^{r}}, \frac{k}{N^{r}}\right] \times\left[\alpha, \alpha+\frac{1}{N^{r}}\right]: k, r \in \mathbb{N}, \alpha \in \mathbb{R}\right\} \text {. }
$$


Let $\mathscr{N}^{*}(r)$ be the minimum number of $\frac{1}{N^{r}} \times \frac{1}{N^{r}}$ squares of $\mathscr{C}$ needed to cover $G$ and let $\mathscr{N}(r)$ be the smallest number of arbitrary $\frac{1}{N^{r}} \times \frac{1}{N^{r}}$ squares which cover $G$. Clearly, $\mathscr{N}^{*}(r) \geqq \mathscr{N}(r)$. Since we can cover any $\frac{1}{N^{r}} \times \frac{1}{N^{r}}$ square in $K$ with two $\frac{1}{N^{r}} \times \frac{1}{N^{r}}$ squares from $\mathscr{C}$ we have $\mathscr{N}^{*}(r) \leqq 2 \mathscr{N}(r)$.

Thus it suffices to look at $\lim _{r \rightarrow \infty} \frac{\log \mathscr{N}^{*}(r)}{\log \left(N^{r}\right)}$.

Theorem. Let $G=\operatorname{graph}(f)$, where $f$ is defined above. If $\sum\left|a_{i}\right|>1$ and the interpolation points $\left(x_{i}, y_{i}\right)$ are not collinear, then $C(G)=1+\log _{N}\left(\sum\left|a_{k}\right|\right)$; otherwise $C(G)=1$.

Proof. Let $C_{r} \in \mathscr{C}$ be a "best" cover of $G$ consisting of $\mathscr{N}^{*}(r) \frac{1}{N^{r}} \times \frac{1}{N^{r}}$ squares whose interiors are disjoint.

Let $C(r, k)$ denote the collection of all the squares in $C_{r}$ which lie between $x=\frac{k-1}{N^{r}}$ and $x=\frac{k}{N^{r}}$. Let $\mathscr{N}(r, k)$ denote the number of squares in $C(r, k)$ and let $\mathscr{R}(r, k)=\bigcup_{\mathscr{A}_{i} \in C(r, k)} \mathscr{A}_{i}$. Since $C_{r}$ is a "best" covering, every square in $C_{r}$ must meet $G$ and since $G$ is the graph of a continuous function, $\mathscr{R}(r, k)$ must be rectangle of width $1 / N^{r}$ and height $\frac{\mathscr{N}(r, k)}{N^{r}}$. Note that $\mathscr{N}^{*}(r)=\sum_{k=1}^{N^{r}} \mathscr{N}(r, k)$. The idea of the proof is to estimate $\mathscr{N}^{*}(r+1)$ in terms of $\mathscr{N}^{*}(r)$.

Now consider $w_{i}(\mathscr{R}(r, k)), i \in\{1, \ldots, N\}$, which is a parallelogram contained in $\left[\frac{l(k, i)-1}{N^{r+1}}, \frac{l(k, i)}{N^{r+1}}\right] \times \mathbb{R}$, where $\frac{l(k, i)}{N^{r+1}}=\frac{k}{N^{r+1}}+\frac{i-1}{N}$. Observe that

$$
\sum_{i=1}^{N} \sum_{k=1}^{N^{r}} \mathscr{N}(r+1, l(k, i))=\mathscr{N}^{*}(r+1) .
$$

Since $G=\bigcup_{i=1}^{N} w_{i}(G)$, we have $G \subset \bigcup_{i=1}^{N} w_{i}\left(\bigcup_{k=1}^{N r} \mathscr{R}(r, k)\right) \cdot w_{i}(\mathscr{R}(r, k))$ is contained in a reactangle of width $1 / N^{r+1}$ and height

$$
\frac{\left|a_{i}\right| \mathcal{N}(r, k)+\left|b_{i}\right|}{N^{r}}
$$

and thus

$$
\begin{aligned}
\mathscr{N}(r+1, l(k, i)) & \leqq\left[\frac{\left|a_{i}\right| \mathcal{N}(r, k)+\left|b_{i}\right|}{N^{r}} / \frac{1}{N^{r+1}}\right]+1 \\
& =N\left[\left|a_{i}\right| \mathcal{N}(r, k)+\left|b_{i}\right|\right]+1
\end{aligned}
$$

Summing over $k$ and $i$ yields $\mathscr{N}^{*}(r+1) \leqq N \gamma \mathscr{N}^{*}(r)+N^{r+1} c_{1}$, where $\gamma=\sum\left|a_{i}\right|$ and $c_{1}=\sum\left|b_{i}\right|+1$. Induction on $r$ gives $\mathscr{N}^{*}(r) \leqq N^{r} \gamma^{r} \mathscr{N}^{*}(1)+c_{1} N^{r}\left(1+\ldots+\gamma^{r-1}\right)$. 
Case 1. $\gamma \leqq 1$ : We then have $\mathcal{N}^{*}(r) \leqq c_{2} r N^{r}$, where $c_{2}=\mathscr{N}^{*}(1)+c_{1}$. Hence

$$
C(G) \leqq \lim _{r \rightarrow \infty} \frac{\ln \left(c_{2} r N^{r}\right)}{\ln N^{r}}=1
$$

Case 2. $\gamma>1$ : We get $\mathscr{N}^{*}(r) \leqq c_{3}(\gamma N)^{r}$, where $c_{3}=\mathscr{N}^{*}(1)+\frac{c_{1}}{1-\gamma}$. Thus

$$
C(G) \leqq \lim _{r \rightarrow \infty} \frac{\ln \left(c_{3}(\gamma N)^{r}\right)}{\ln N^{r}}=1+\log _{N} \gamma .
$$

We will now find a lower bound for $C(G)$. Since $G$ is the graph of a continuous function, we have $C(G) \geqq 1$. Hence if $\gamma \leqq 1$, we have $C(G)=1$.

If all the interpolation points lie on a common line $L$, then $G=L$ and so $C(G)=1$. Suppose then that $\gamma>1$ and not all interpolation points lie on a common line. We need the following result.

Lemma. If $\gamma>1$ and not all interpolation points lie on a common line, then

$$
\lim _{r \rightarrow \infty} \frac{\mathscr{N}^{*}(r)}{N^{r}}=\infty
$$

Proof. Since all the interpolation points do not lie on a common line there exists a $j \in\{1, \ldots, N\}$ such that

$$
V=\left|y_{j}-\left(y_{N}-y_{0}\right) x_{j}-y_{0}\right|>0
$$

(see Fig. 1). Clearly

$$
V \leqq \max \left\{\left|y_{j}-y_{0}\right|,\left|y_{j}-y_{N}\right|\right\} \text {. }
$$

Since $G$ is the graph of a continuous function, we have $\mathscr{N}^{*}(r) \geqq\left[V N^{r}\right]$. Also $\mathscr{N}^{*}(r)$ $\geqq \sum_{i=1}^{N}\left[\left|a_{i}\right| V N^{r}\right]$ for $r \geqq 1$ (see Fig. 2). By induction

$$
\mathscr{N}^{*}(r) \geqq \sum_{i_{1}=1}^{N} \sum_{i_{k}=1}^{N}\left[\left|a_{i_{1}} \ldots a_{i_{k}}\right| V N^{r}\right] \quad \text { for } \quad r \geqq k,
$$

and thus $\mathscr{N}^{*}(r) \geqq\left(V\left(\sum_{i=1}^{N}\left|a_{i}\right|\right)^{r}-1\right) N^{r}$.

Fig. 1

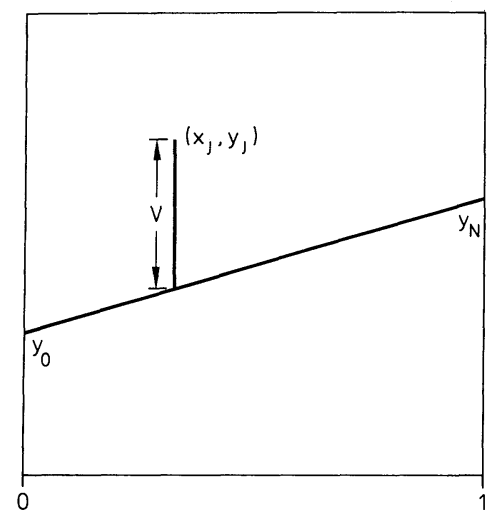

Fig. 2

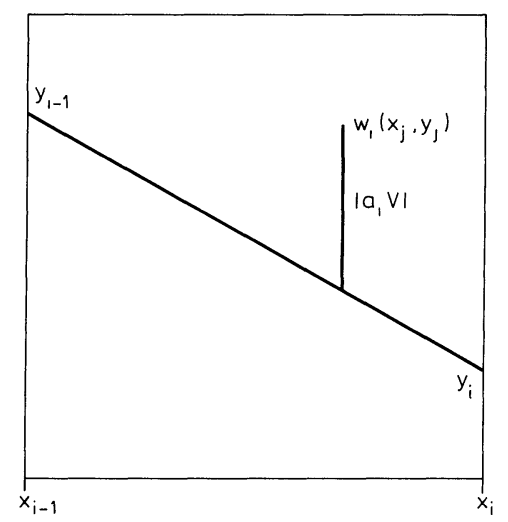


Since each $\mathscr{A}_{i} \in C(r, k)$ meets $G$, each $w_{i}\left(\mathscr{A}_{i}\right), i \in\{1, \ldots, N\}$ must also meet $G$ and so $C(r+1, l(i, k))$ must at least cover a rectangle of height

Thus

$$
\frac{\left|a_{i}\right|(\mathscr{N}(r, k)-2)-\left|b_{i}\right|}{N^{r}} .
$$

$$
\mathscr{N}(r+1, l(i, k)) \geqq N\left(\left|a_{i}\right|(\mathscr{N}(r, k)-2)-\left|b_{i}\right|\right)-1 .
$$

Summing over $k$ and $i$ yields $\mathscr{N}^{*}(r+1) \geqq(N \gamma) \mathscr{N}^{*}(r)-c_{4} N^{r+1}$, where $c_{4}=1+\sum\left(2\left|a_{i}\right|+\left|b_{i}\right|\right)$. Induction on $r$ gives

$$
\mathscr{N}^{*}(r+1) \geqq(\gamma N)^{r-l-1}\left(\mathscr{N}^{*}(l-1)-\frac{c_{4} N^{l}}{1-\gamma}\right)
$$

for all $1 \leqq l \leqq r$. Note that we can choose $l$ large enough to ensure

$$
\mathscr{N}^{*}(l-1)-\frac{c_{4} N^{l}}{1-\gamma}>0 .
$$

Set

$$
c_{5}=\gamma N^{-l-1}\left(\mathscr{N}^{*}(l-1)-\frac{c_{4} N}{1-\gamma}\right)>0 .
$$

Then $\mathscr{N}^{*}(r) \geqq c_{5}(\gamma N)^{r}$ and thus $C(G) \geqq 1+\log _{N} \gamma$.

\section{Remarks}

We can construct a dynamical system $(M, W, v)$ associated with the h.i.f.s. $\left(K, w_{i}: i=1, \ldots, N\right)$ the following way: $M=K \times[0,1], \quad v=\mu \times m$ ( $m$ onedimensional Lebesgue measure on $[0,1])$ and $W: M \gg$,

$$
W(x, y)=\left(w_{i}(x), \frac{y-s_{i-1}}{s_{i}}\right) \text { if } y \in\left[s_{i}, s_{i-1}\right],
$$

where $s_{i}=p_{1}+\ldots+p_{i}$ for $i=1, \ldots, N$ and $s_{0}=0$ (for similar constructions see [Ba, FaOtYo, Pe]). Using the maps $w_{i}, i=1, \ldots, N$, from Sect. II a straightforward calculation gives the Lyapunov exponents

$$
\lambda_{1}=\sum_{i=1}^{N} p_{i} \log \frac{1}{p_{i}}, \quad \lambda_{2}=\sum_{i=1}^{N} p_{i} \log \left|a_{i}\right|,
$$

and

$$
\lambda_{3}=\log \frac{1}{N}
$$

for this dynamical system. The Lyapunov dimension of $v$ is then given by

$$
\Lambda(v, p)=2+\sum p_{i} \log _{N} \frac{\left|a_{i}\right|}{p_{i}}
$$

provided that $\lambda_{2}>\lambda_{3}$; otherwise

$$
\Lambda(v, p)=1+\sum p_{i} \log _{N}\left(1 / p_{i}\right) .
$$


Maximizing over the probabilities $p_{i}$ yields

$$
\Lambda=2+\log _{N}\left(\sum_{i=1}^{N}\left|a_{i}\right|\right)
$$

for $\sum_{i=1}^{N}\left|a_{i}\right|>1$ and $\Lambda=2$ otherwise.

Notice that the capacity of the attractor for the dynamical system $(M, W, v)$ is $1+C(G)$ and that $\Lambda-1=C(G)$ if the interpolation points are not collinear. This confirms a conjecture of Yorke's in this particular case (see [FrKaYo]).

Acknowledgements. We would like to thank our thesis advisor Prof. Barnsley who showed us the connections between i.f.s. and dynamical systems and who gave us considerable insight into this problem. We are also grateful to Prof. Harrington and Prof. Geronimo for helpful discussions.

\section{References}

[Mo] Moran, P.A.P.: Additive functions of intervals and Hausdorff measure. Proc. Camb. Phil. Soc. 42, 15-23 (1946)

[Hu] Hutchinson, J.: Fractals and self-similarity. Indiana Univ. Math. J. 30, 5, 713-747 (1981)

[BaDe] Barnsley, M.F., Demko, S.: Iterated function systems and the global construction of fractals. Proc. R. Soc. Lond. A399, 243-275 (1985)

[BaHa] Barnsley, M.F., Harrington, A.N.: The calculus of fractal interpolation functions. Georgia Tech. preprint (1985)

[BeUr] Besicovitch, A.S., Ursell, H.D.: Sets of fractional dimensions, V: On dimensional numbers of some continuous curves. J. Lond. Math. Soc. 12, 18-25 (1937)

[KaMaYo] Kaplan, J.L., Mallet-Paret, J., Yorke, J.A.: The Lyapunov dimension of a nowhere differentiable attracting torus. Ergodic Theory Dyn. Syst. 4, 261-281 (1984)

[Ba] Barnsley, M.F.: Fractal functions and interpolation. Georgia Tech. Preprint (1985)

[FaOtYo] Farmer, J.D., Ott, E., Yorke, J.A.: The dimension of chaotic attractors. Physica 7D, 153-180 (1983)

[Pe] Pelikan, S.: Invariant densities for random maps of the interval. Trans. Am. Math. Soc. 281 (2), 313-325

[FrKaYo] Frederickson, P., Kaplan, J.L., Yorke, E.D., Yorke, J.A.: The Lyapunov dimension of strange attractors. J. Differ. Equations 49, 185-207 (1983)

Communicated by J.-P. Eckmann

Received October 15, 1985; in revised form February 12, 1986 